\section{SOI: $1.1 /$ TAS $\quad$ DOI: $10.15863 /$ TAS \\ International Scientific Journal Theoretical \& Applied Science}

p-ISSN: 2308-4944 (print) e-ISSN: 2409-0085 (online)

Year: 2018 Issue: 02 Volume: 58

Published: 19.02.2018 http://T-Science.org

SECTION 9: Chemistry and chemical technology
Oleg Ivanovych Yurchenko Kharkiv V.N. Karazine National University, PhD, Full Professor of Chemical Metrology Department, yurchenko@karazin.ua

Nadegda Petrovna Titova Kharkiv V.N. Karazin

National University, Researcher of Chemical Metrology Department yurchenko@karazin.ua

Konstantin Nikolayevich Belikov Acting Deputy General Director Scientific Institution "Institute for Single Crystals" of National Academy of Sciences of Ukraine belikov@isc.kharkov.com

Tetyana Vasylivna Chernozhuk Kharkiv V.N.

Karazine National University, PhD, Associate Professor of Inorganic Chemistry Department, tanya.chernozhuk@gmail.com

Oleksii Andriovych Kravchenko Kharkiv V.N.

Karazine National University, PhD, Associate Professor of Chemical Metrology Department alekseykravch@ukr.net

Tetana Sergiivna Tatarina Kharkiv V.N. Karazine

National University, student of Chemical Metrology Department, yurchenko@karazin.ua

\title{
ATOMIC-ABSORPTION AND ATOMIC-EMISSION WITH INDUCTIVE CONNECTED PLASMA DETERMINATION OF IRON AND MANGANESE IN CURATIVE CLAYS
}

\footnotetext{
Abstract: Atomic-absorption and atomic-emission with inductive connected plasma determination of Iron and Manganese in curative clays was carried out. It was shown that maximum of analytical signal is getting at using triton $X-100(\omega=4 \%)$ and ultrasound treatment of the analyzed solutions during 20 minutes. By variation of the sample it was established, that systematic error is ambiguous. An accuracy of the results of analysis was checked by the method "injected-found out". Coherence of the results, obtained by two independent methods was estimated by $F$-and $t$-criteria. It was proved that run of the means is not sufficient and proved by random scatter. Detection limit of Iron and Manganese was estimated.

Key words: Manganese, Iron, ultrasound treatment, atomic-absorption and atomic-emission with inductive connected plasma spectrometry, green clay, triton X-100, metrological characteristics.

Language: English

Citation: Yurchenko OI, Titova NP, Belikov KN, Chernozhuk TV, Kravchenko OA, Tatarina TS (2018) ATOMIC-ABSORPTION AND ATOMIC-EMISSION WITH INDUCTIVE CONNECTED PLASMA DETERMINATION OF IRON AND MANGANESE IN CURATIVE CLAYS. ISJ Theoretical \& Applied Science, 02 (58): 53-59.

Soi: http://s-o-i.org/1.1/TAS-02-58-14 Doi: crossef https://dx.doi.org/10.15863/TAS.2018.02.58.14
} 


\section{Introduction}

The term "clay" unite quite wide class of rocks of drag nature. It consists of tiny particles of minerals that formed as a result of wind and aqua erosion. Chemical composition of clay is determined by composition of these rocks and is different on various territories. Therefore, clays, mined at different territories, are different too. Because of clays are formed from the compounds of earth crust, its chemical composition is similar: silicates of aluminum, potassium cations, sodium, magnesium, calcium, so on.

Formation of clay is quite slow process: tiny particles of dust, settled on soil surface, penetrate through gravel, sand and in filtration process stick together, forming thin layer, that does not conduct water and stop movement of such particles. So, in such way formation of clay layer ( $1 \mathrm{~mm}$ per 3 years) begun.

Green clay contains all of the mineral salts and microelements what we need: Silex, Phosphate, Ferum, Calcium, Magnesium, Potassium etc., and in good assimilable form for human organism. [1,p.200; 2,p.134; 3,p.245; 4,p.254; 5,p.24; 6,p.3].

Express methods of spectral atomic analysis (SAS) is widely used in industry, agriculture, geology and another brunches of science and economy.

Methods of atomic-absorption and atomicemission with inductive connected plasma spectrometry are universal at analytes determination in many components systems [7,p.596; 8,p.27; 9,p.34; 10,p.152; 11,p.97;12.p.432].

The purpose of the work is to determine analytes by atomic-absorption and atomic-emission with inductive connected plasma spectrometry in the samples of curative clays, using modern methods of samples preparation.

\section{Experimental part}

An analysis of green clay samples to find out Iron and Manganese was done by atomic-absorption spectrometer C-115-MI and by atomic-emission with inductive connected plasma spectrometer Trace SCAN Thermo Jarrell Ach (USA). For sample preparation an ultrasound bath PS-20 was used. Substances of c.p. qualification were used, triton X$100(\omega=4 \%)$ standard samples, based on water solutions of metals acytylacetonates with concentration of Iron and Manganese $0,1 \mathrm{gll}$ (acytylacetonates of Iron and Manganese metals is used as standard ones by a lot of Ukrainian factories). The object of investigation was green clay from Luzhok village, Krahkiv region.

To build calibrated graphs 0,$2 ; 0,6 ; 1,0 ; 1,4$; $2,0 \mathrm{ml}$ of initial solution was put into 5 volumetric flasks of $10 \mathrm{ml}$ volume, $6 \mathrm{ml}$ of Triton X-100 ( $\omega=4$ $\%$ ) was added to it. It was made up by distilled water and mixed. The obtained solutions contains $1 * 10^{-4}$, $3^{*} 10^{-4}, 5^{*} 10^{-4}, 7 * 10^{-4}, 10 * 10^{-4} \mathrm{~g} / 1$ Iron or Manganese correspondingly.

For analysis were taken samples from 0,1 to 0,5 $\mathrm{g}$, scaled on electronic scales PA-64. 2,5 ml of saturated $\mathrm{HNO}_{3}$ was added to the glasses and samples were dissolved at heating. To the wet precipitate 2,5 $\mathrm{ml}$ of $1,5 \% \mathrm{HNO}_{3}, 6 \mathrm{ml}$ of Triton $\mathrm{X}-100$ were added and it was put in the flask of $10 \mathrm{ml}$ volume. It was made up by acetylacetone and mixed.

\section{Results and discussion}

To prepare calibrated solutions, based on SAS, choice of Triton X-100 concentrations was carried out (table1).

According to the obtained data, it was found out that Triton X-100 with $(\omega=4 \%)$ makes maximal analytical signal of Iron and Manganese in calibrated solutions.

Choice of optimal time of ultrasound treatment of calibrated solutions is in the table 2 .

According to the data from the table, we choose time of ultrasound treatment about 20 minutes.

Calibrated graphs of atomic-absorption determination of Iron and Manganese are on pics 1,2

It was shown that using of Tritone X-100 $(\omega=4$ $\%$ ) increase sensibility of atomic-absorption determination of Iron and Manganese in 1,4 times.

Variation of mass of the samples of green clay to found out systematic error was done. (table 3).

Atomic-absorption and atomic-emission with inductive connected plasma determination of Iron and Manganese in analyzed samples was carried out. (tables 4,5)

By "injected-found out" method verification of accuracy of atomic-absorption determination of analytes was done. (table 6)

It was shown that the results has no systematic errors.

Comparison of the results, obtained by two independent methods was done. (table 7)

It was shown that methodic has no sufficient systematic errors, and dispersion of the results is proved at random.

The limit of atomic-absorption determination of analytes in the analyzed solutions was estimated. To do it 20 blank solutions were prepared and analytical signal was measured for it. Calculations are in tables 8,9 .

It was shown that found out value of $\mathrm{C}_{\min }$ is lower than literature one. [13,p.178]

\section{Conclusions}

Using of acetylacetone to exctract Iron and Manganese and acetylacetone to calibrate devises leads to identity of analyzed and calibrated solutions. 


\begin{tabular}{|c|c|c|c|c|c|c|}
\hline Impact Factor: & $\begin{array}{l}\text { ISRA (India) } \\
\text { ISI (Dubai, UAE } \\
\text { GIF (Australia) } \\
\text { JIF }\end{array}$ & $\begin{array}{l}=1.344 \\
=0.829 \\
=0.564 \\
=1.500\end{array}$ & $\begin{array}{l}\text { SIS (USA) } \\
\text { PИНЦ (Russia) } \\
\text { ESJI (KZ) } \\
\text { SJIF (Morocco) }\end{array}$ & $\begin{array}{l}=0.912 \\
=0.207 \\
=4.102 \\
=2.031\end{array}$ & $\begin{array}{l}\text { ICV (Poland) } \\
\text { PIF (India) } \\
\text { IBI (India) }\end{array}$ & $\begin{array}{l}=6.630 \\
=1.940 \\
=4.260\end{array}$ \\
\hline
\end{tabular}

The fact gives us possibility to decrease systematic error. So, using of Tritone X-100 $(\omega=4 \%)$ and ultrasound treatment during 20 minutes also using of acetylacetonates of Iron and Manganese let us to extract analytes from the analyzed samples totally. Limit of determination of the analytes is less than literature one.

Choice of Triton X-100 concentrations

Table 1

\begin{tabular}{|c|c|c|c|c|}
\hline$\omega, \%$ & $\mathrm{Mn}, \mathrm{mg} / \mathrm{kg}$ & $\mathrm{S}_{\mathrm{r}}$ & $\mathrm{Fe}, \mathrm{g} / \mathrm{kg}$ & $\mathrm{S}_{\mathrm{r}}$ \\
\hline 2 & $20,07 \pm 0,25$ & 0,01 & $7,20 \pm 0,18$ & 002 \\
\hline 3 & $21,12 \pm 0,26$ & 0,01 & $7,66 \pm 0,19$ & 0,02 \\
\hline 4 & $22,24 \pm 0,28$ & 0,01 & $8,06 \pm 0,10$ & 0,01 \\
\hline 5 & $22,10 \pm 0,27$ & 0,01 & $8,01 \pm 0,10$ & 0,01 \\
\hline 6 & $21,85 \pm 0,27$ & 0,01 & $7,92 \pm 0,10$ & 0,01 \\
\hline
\end{tabular}

Table 2

Choice of time of ultrasound treatment

\begin{tabular}{|c|c|c|c|c|}
\hline T, min. & $\mathrm{Mn}, \mathrm{mg} / \mathrm{kg}$ & $\mathrm{S}_{\mathrm{r}}$ & $\mathrm{Fe}, \mathrm{g} / \mathrm{kg}$ & $\mathrm{S}_{\mathrm{r}}$ \\
\hline 10 & $20,57 \pm 0,26$ & 0,01 & $7,45 \pm 0,09$ & 0,01 \\
\hline 15 & $21,54 \pm 0,27$ & 0,01 & $7,80 \pm 0,10$ & 0,01 \\
\hline 20 & $22,24 \pm 0,28$ & 0,01 & $8,06 \pm 0,10$ & 0,01 \\
\hline 25 & $22,06 \pm 0,27$ & 0,01 & $7,99 \pm 0,10$ & 0,01 \\
\hline
\end{tabular}

Statistics, deals with the results of analysis

Table 3

\begin{tabular}{|c|c|c|}
\hline $\mathrm{Y}=\mathrm{A}+\mathrm{B} * \mathrm{X}$ & $\mathrm{I}\left(\mathrm{A}_{\text {water }}\right)$ & II $\left(\mathrm{A}_{\text {modif.. }}\right)$ \\
\hline $\mathrm{A}$ & 0.082 & 0.041 \\
\hline $\mathrm{B}$ & 51311 & 61844 \\
\hline Number of points & 5 & 5 \\
\hline Correlation coefficient & 0,9997 & 0,9997 \\
\hline Residual dispersion & 0.25 & 0.25 \\
\hline Dispersion & 0.00084 & 0.00084 \\
\hline
\end{tabular}

Table 4

Variation of mass of the samples of green clay

\begin{tabular}{|c|c|c|c|c|}
\hline \multirow{2}{*}{$\mathrm{m}, \mathrm{g}$} & \multicolumn{2}{|c|}{$\mathrm{Mn}, \mathrm{mg} / \mathrm{l}$} & \multicolumn{2}{c|}{$\mathrm{Fe}, \mathrm{mg} / \mathrm{l}$} \\
\cline { 2 - 5 } & $\bar{c} \pm \frac{t_{p . f} \times S}{\sqrt{n}}$ & $\mathrm{~S}_{\mathrm{r}}$ & $\bar{c} \pm \frac{t_{p . f} \times S}{\sqrt{n}}$ & \\
\hline 0,1 & $0,055 \pm 0,004$ & 0,01 & $0,15 \pm 0,01$ & 0,04 \\
\hline 0,2 & $0,11 \pm 0,01$ & 0,04 & $0,29 \pm 0,01$ & 0,03 \\
\hline 0,3 & $0,16 \pm 0,01$ & 0,04 & $0,44 \pm 0,01$ & 0,02 \\
\hline 0,4 & $0,22 \pm 0,01$ & 0,04 & $0,59 \pm 0,01$ & 0,01 \\
\hline 0,5 & $0,27 \pm 0,01$ & 0,03 & $0,73 \pm 0,01$ & 0,01 \\
\hline \hline
\end{tabular}




\begin{tabular}{|c|c|c|c|c|c|c|}
\hline Impact Factor: & $\begin{array}{l}\text { ISRA (India) } \\
\text { ISI (Dubai, UAE } \\
\text { GIF (Australia) } \\
\text { JIF }\end{array}$ & $\begin{array}{l}=1.344 \\
=0.829 \\
=0.564 \\
=1.500\end{array}$ & $\begin{array}{l}\text { SIS (USA) } \\
\text { PИНЦ (Russia) } \\
\text { ESJI (KZ) } \\
\text { SJIF (Morocco) }\end{array}$ & $\begin{array}{l}=0.912 \\
=0.207 \\
=4.102 \\
=\mathbf{2 . 0 3 1}\end{array}$ & $\begin{array}{l}\text { ICV (Poland) } \\
\text { PIF (India) } \\
\text { IBI (India) }\end{array}$ & $\begin{array}{l}=6.630 \\
=1.940 \\
=4.260\end{array}$ \\
\hline
\end{tabular}

Table 5

The results of Iron and Manganese determination by AAC method in green clay $(\mathrm{n}=5, \mathrm{P}=0.95)$

\begin{tabular}{|c|c|c|c|c|}
\hline \multirow{2}{*}{ Sample } & \multicolumn{2}{|c|}{$\mathrm{Mn}, \mathrm{mg} / \mathrm{kg}$} & \multicolumn{2}{c|}{$\mathrm{Fe}, \mathrm{mg} / \mathrm{kg}$} \\
\cline { 2 - 4 } & $\bar{c} \pm \frac{t_{p . f} \times S}{\sqrt{n}}$ & $\mathrm{~S}_{\mathrm{r}}$ & $\bar{c} \pm \frac{t_{p . f} \times S}{\sqrt{n}}$ & $\mathrm{~S}_{\mathrm{r}}$ \\
\hline Green clay & $22,24 \pm 0,28$ & & $8060 \pm 100$ & 0,01 \\
\hline
\end{tabular}

Table 6

The results of Iron and Manganese determination by AEC-IZP method in green clay $(\mathrm{n}=5, \mathrm{P}=\mathbf{0 . 9 5})$

\begin{tabular}{|c|c|c|c|c|}
\hline \multirow{2}{*}{ Sample } & \multicolumn{2}{|c|}{$\mathrm{Mn}, \mathrm{mg} / \mathrm{kg}$} & \multicolumn{2}{c|}{$\mathrm{Fe}, \mathrm{mg} / \mathrm{kg}$} \\
\cline { 2 - 4 } & $\bar{c} \pm \frac{t_{p . f} \times S}{\sqrt{n}}$ & $\mathrm{~S}_{\mathrm{r}}$ & $\bar{c} \pm \frac{t_{p . f} \times S}{\sqrt{n}}$ & $\mathrm{~S}_{\mathrm{r}}$ \\
\hline Green clay & $22,24 \pm 0,28$ & & $8060 \pm 105$ & 0,01 \\
\hline
\end{tabular}

Table 7

Verification of accuracy of atomic-absorption determination of Iron and Manganese by "injected-found out" method $(n=5, P=0.95)$

\begin{tabular}{|c|c|c|c|c|}
\hline & Content & Injected & Found out & $\mathrm{S}_{\mathrm{r}}$ \\
\hline $\mathrm{Fe}, \mathrm{mg} / \mathrm{kg}$ & 8060 & 8000 & $1610 \pm 199$ & 0.01 \\
\hline $\mathrm{Mn}, \mathrm{mg} / \mathrm{kg}$ & 22.24 & 20.0 & $42.26 \pm 0.52$ & 0.01 \\
\hline
\end{tabular}

Table 8

Comparison of the results of Iron and Manganese determination by AAC and AEC-I3П methods in green clay, stabilized by US treatment, according to Fisher and Student criteria.

\begin{tabular}{|c|c|c|c|}
\hline Metal & $\mathrm{F}$ & $\mathrm{S}_{1.2}$ & $\mathrm{t}_{1.2}$ \\
\hline Manganese & 1,57 & 0,064 & 1,25 \\
\hline Iron & 1,02 & 0,007 & 1,95 \\
\hline
\end{tabular}

At $n=5, p=0,95$

$\begin{array}{ll}\mathrm{F}_{\text {table }}=6,39 & \mathrm{~F}<\mathrm{F}_{\text {table }} \\ \mathrm{t}_{\text {table }}=2,31 & \mathrm{t}<\mathrm{t}_{\text {table }}\end{array}$




\begin{tabular}{|c|c|c|c|c|c|c|}
\hline Impact Factor: & $\begin{array}{l}\text { ISRA (India) } \\
\text { ISI (Dubai, UAF } \\
\text { GIF (Australia) } \\
\text { JIF }\end{array}$ & $\begin{array}{l}=1.344 \\
=0.829 \\
=0.564 \\
=1.500\end{array}$ & $\begin{array}{l}\text { SIS (USA) } \\
\text { PИНЦ (Russia) } \\
\text { ESJI (KZ) } \\
\text { SJIF (Morocco) }\end{array}$ & $\begin{array}{l}=0.912 \\
=0.207 \\
=4.102 \\
=2.031\end{array}$ & $\begin{array}{l}\text { ICV (Poland) } \\
\text { PIF (India) } \\
\text { IBI (India) }\end{array}$ & $\begin{array}{l}=6.630 \\
=1.940 \\
=4.260\end{array}$ \\
\hline
\end{tabular}

Table 9

An estimation of limit of determination of Manganese $C_{\min }(\mathrm{mg} / \mathrm{l})$ in clay by atomic-absorption method.

\begin{tabular}{|c|c|c|c|c|c|c|c|c|c|}
\hline № & $\mathrm{A}_{1}$ & $\mathrm{~A}_{2}$ & $\mathrm{~A}_{3}$ & $\mathrm{~A}_{4}$ & $\mathrm{~A}_{5}$ & $\mathrm{~A}_{6}$ & $A_{c p}$ & $\mathrm{~S}_{0}$ & $\mathrm{C}_{\min }$ \\
\hline 1 & 3 & 6 & 1 & 5 & 1 & 4 & 3.2 & 0.23 & 0.003 \\
\hline 2 & 3 & 2 & 3 & 2 & 3 & 2 & 2.5 & \multirow{10}{*}{$\mathrm{C}_{\mathrm{lit}}=0,004$} & \\
\hline 3 & 4 & 3 & 5 & 1 & 3 & 2 & 3.0 & & \\
\hline 4 & 4 & 3 & 2 & 3 & 2 & 3 & 2.8 & & \\
\hline 5 & 1 & 6 & 3 & 5 & 4 & 1 & 3.2 & & \\
\hline 6 & 3 & 2 & 3 & 2 & 3 & 2 & 2.5 & & \\
\hline 7 & 2 & 3 & 1 & 5 & 3 & 4 & 3.0 & & \\
\hline 8 & 3 & 4 & 3 & 2 & 3 & 2 & 2.8 & & \\
\hline 9 & 3 & 5 & 1 & 4 & 6 & 1 & 3.2 & & \\
\hline 10 & 3 & 4 & 1 & 5 & 2 & 3 & 3.0 & & \\
\hline 11 & 2 & 3 & 2 & 3 & 4 & 2 & 2.8 & & \\
\hline 12 & 3 & 2 & 3 & 2 & 3 & 2 & 2.5 & & \\
\hline 13 & 5 & 3 & 1 & 6 & 1 & 4 & 3.2 & & \\
\hline 14 & 2 & 3 & 1 & 5 & 3 & 4 & 3.0 & & \\
\hline 15 & 4 & 3 & 2 & 3 & 2 & 3 & 2.8 & & \\
\hline 16 & 2 & 3 & 2 & 3 & 2 & 3 & 2.5 & & \\
\hline 17 & 4 & 1 & 6 & 1 & 5 & 3 & 3.2 & & \\
\hline 18 & 3 & 2 & 3 & 2 & 3 & 2 & 2.5 & & \\
\hline 19 & 4 & 3 & 5 & 1 & 3 & 2 & 3.0 & & \\
\hline 20 & 2 & 3 & 4 & 3 & 2 & 3 & 2.8 & & \\
\hline
\end{tabular}




\begin{tabular}{|c|c|c|c|c|c|c|}
\hline Impact Factor: & $\begin{array}{l}\text { ISRA (India) } \\
\text { ISI (Dubai, UAF } \\
\text { GIF (Australia) } \\
\text { JIF }\end{array}$ & $\begin{array}{l}=1.344 \\
=0.829 \\
=0.564 \\
=1.500\end{array}$ & $\begin{array}{l}\text { SIS (USA) } \\
\text { PИНЦ (Russia) } \\
\text { ESJI (KZ) } \\
\text { SJIF (Morocco) }\end{array}$ & $\begin{array}{l}=0.912 \\
=0.207 \\
=4.102 \\
=2.031\end{array}$ & $\begin{array}{l}\text { ICV (Poland) } \\
\text { PIF (India) } \\
\text { IBI (India) }\end{array}$ & $\begin{array}{l}=6.630 \\
=1.940 \\
=4.260\end{array}$ \\
\hline
\end{tabular}

Table 10

An estimation of limit of determination of $\operatorname{Iron} \mathrm{C}_{\min }(\mathrm{mg} / \mathrm{l})$ in clay by atomic-absorption method.

\begin{tabular}{|c|c|c|c|c|c|c|c|c|c|}
\hline № & $\mathrm{A}_{1}$ & $\mathrm{~A}_{2}$ & $\mathrm{~A}_{3}$ & $\mathrm{~A}_{4}$ & $\mathrm{~A}_{5}$ & $\mathrm{~A}_{6}$ & $\mathrm{~A}_{\mathrm{cp}}$ & $\mathrm{S}_{0}$ & $\mathrm{C}_{\text {min }}$ \\
\hline 1 & 2 & 4 & 2 & 3 & 1 & 2 & 2.33 & 0.19 & 0.014 \\
\hline 2 & 1 & 3 & 1 & 2 & 3 & 2 & 2.0 & \multirow{10}{*}{$\mathrm{C}_{\mathrm{lit}}=0,015$} & \\
\hline 3 & 3 & 2 & 3 & 2 & 3 & 2 & 2.5 & & \\
\hline 4 & 2 & 1 & 2 & 3 & 1 & 2 & 1.83 & & \\
\hline 5 & 2 & 4 & 2 & 3 & 1 & 2 & 2.33 & & \\
\hline 6 & 1 & 3 & 1 & 2 & 3 & 2 & 2.0 & & \\
\hline 7 & 2 & 3 & 2 & 3 & 2 & 3 & 2.5 & & \\
\hline 8 & 2 & 1 & 2 & 3 & 1 & 2 & 1.83 & & \\
\hline 9 & 2 & 4 & 2 & 3 & 1 & 2 & 2.33 & & \\
\hline 10 & 1 & 3 & 1 & 2 & 3 & 2 & 2.0 & & \\
\hline 11 & 2 & 3 & 2 & 3 & 2 & 3 & 2.5 & & \\
\hline 12 & 2 & 1 & 3 & 2 & 1 & 1 & 1.83 & & \\
\hline 13 & 1 & 3 & 1 & 2 & 3 & 2 & 2.0 & & \\
\hline 14 & 2 & 4 & 2 & 3 & 1 & 2 & 2.33 & & \\
\hline 15 & 2 & 3 & 2 & 3 & 2 & 2 & 2.5 & & \\
\hline 16 & 2 & 3 & 2 & 1 & 2 & 1 & 1.83 & & \\
\hline 17 & 3 & 2 & 1 & 2 & 1 & 3 & 1.83 & & \\
\hline 18 & 3 & 2 & 3 & 2 & 3 & 1 & 2.5 & & \\
\hline 19 & 2 & 1 & 3 & 2 & 4 & 2 & 2.33 & & \\
\hline 20 & 2 & 3 & 2 & 1 & 3 & 3 & 2.0 & & \\
\hline
\end{tabular}




\begin{tabular}{l|lr|ll|ll} 
& ISRA (India) & $=\mathbf{1 . 3 4 4}$ & SIS (USA) & $=\mathbf{0 . 9 1 2}$ & ICV (Poland) & $=\mathbf{6 . 6 3 0}$ \\
Impact Factor: & ISI (Dubai, UAE) $=\mathbf{0 . 8 2 9}$ & PUHL (Russia) $=\mathbf{0 . 2 0 7}$ & PIF (India) & $=\mathbf{1 . 9 4 0}$ \\
& GIF (Australia) & $=\mathbf{0 . 5 6 4}$ & ESJI (KZ) & $=\mathbf{4 . 1 0 2}$ & IBI (India) & $\mathbf{4 . 2 6 0}$ \\
& JIF & $=\mathbf{1 . 5 0 0}$ & SJIF (Morocco) & $=\mathbf{2 . 0 3 1}$ & & \\
\hline
\end{tabular}
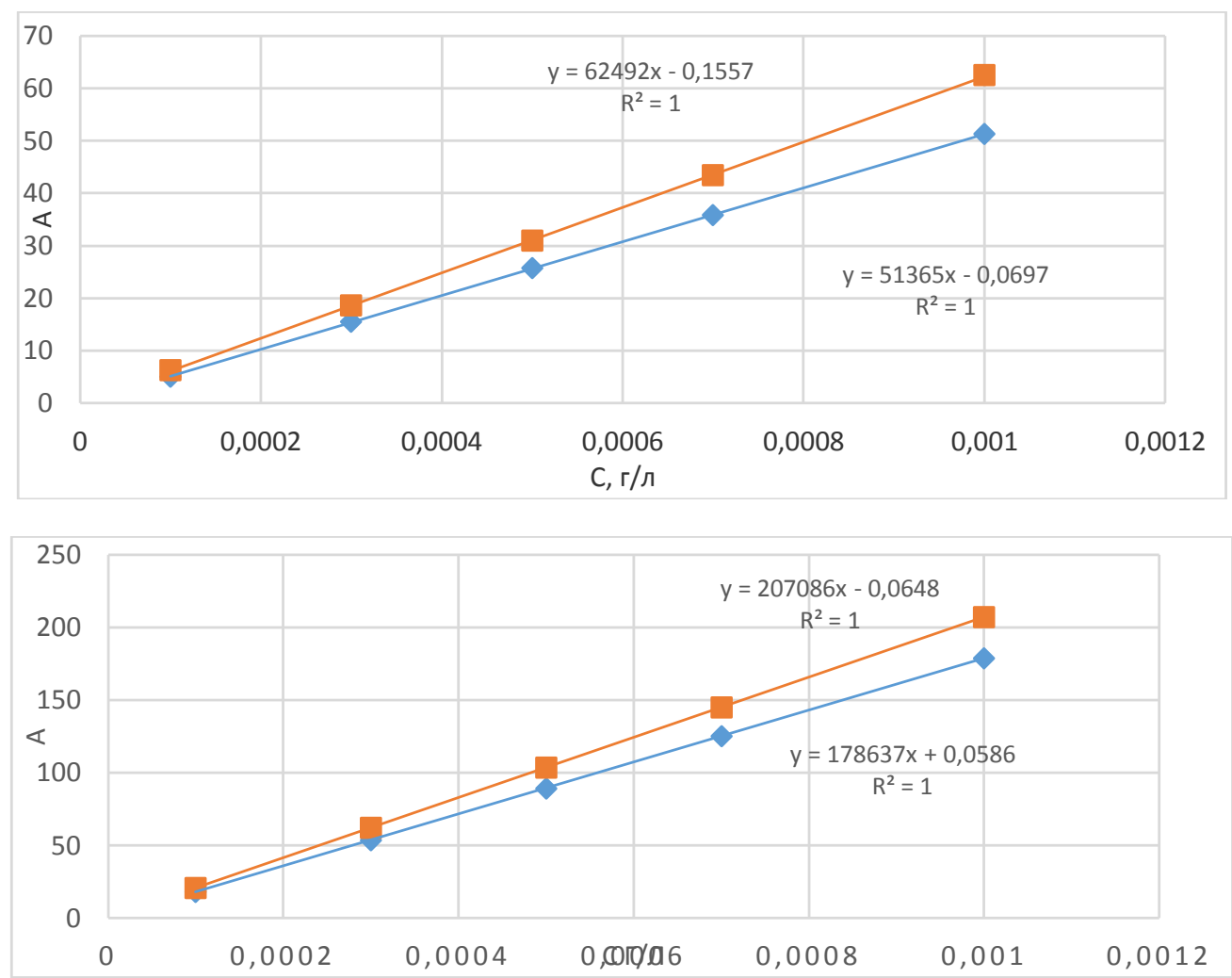

Pic. 1,2 - Calibrated graphs for water solutions of Iron and solutions of Iron, based on Triton X-100 ( $\omega=4 \%)$.

\section{References:}

1. Romanutin A.A., Nazarevyc R.R.. (1995) Medicina Ukrainy. V.2 .p.200- 205.

2. Zhovinskii E. Ya. (2002) Geochemistry of rare metals in Ukrainian soils. Kyiv, Naukova dumka, 2002, $214 \mathrm{p}$.

3. Fateev A.I., Samohvalova V.L. (2008) Naukovy visnyk Uzhorodskogo universitety. V. 22. p.143- 151

4. Kalibina L.V., Nabyvanets B.J. (1996) Analytical chemistry on Enviroment Kyiv, Lybid, 1996, 304 p.

5. Tchekman I.S., Ovrutskii V.I, Shumeiko V.M. (1991) Farmacevtychy zhurnal.V. 1.pp 22-25.

6. (2006) DSTU 4287:2004. 2005.. p.5

7. Ahmed Hassan, A. Jamal Mayouf (2009) American Journal of Applied Sciences V.6. - p. $594-600$
8. Volynsky A.B. (2005) Ukrainian chimichny zhurnal V.9. - p. 25-31

9. Pupushev A.A. (2009) Atomic absorption spectral analysis. Moscow, Technosphera, 2009, $55 \mathrm{p}$.

10. Barsukov V.I. (2004) Fire-emission and atomicabsorption methods of analysis and tool ways to increase its sensibility. Moscow, Mashinostroenie, 2004, 172 p.

11. Yurchenko O.I. (2010) Visnyk Kharkivskogo Nationalnogo universitety. V.18(41).pp 93-100

12. Beckhoff B., Kanngiesser B. (2000) .Handbook of Practical X-Ray Florescence Anaiysis. Berlin, Springer, 2000, $863 \mathrm{p}$.

13. Alemasova A.N. (2003) Analytical atomicabsorption spectroscopy. Sevastopol, Veber, 2003, 308 p. 